García, C. (2018). Los físicos teóricos debaten y ayudan a los teóricos de la contabilidad. Contaduría Universidad de Antioquia, 72, 29-38

Doi: https://doi.org/10.17533/udea.rc.n72a02

\title{
Los físicos teóricos debaten y ayudan a los teóricos de la contabilidad
}

Carlos Luis García Casella

ceconta@fce.uba.ar

Universidad de Buenos Aires 


\section{Los físicos teóricos debaten y ayudan a los teóricos de la contabilidad}

Resumen: El presente trabajo reflexiona sobre el estadio actual de los fundamentos de la teoría contable y el reconocimiento a Max Planck, Albert Einstein y James Murphy sobre sus abordajes metodológicos para las ciencias sociales, en una reflexión sobre la ayuda que pueden representar para los teóricos de la contabilidad. Frente a estos textos, consideramos necesario que los teóricos de la Contabilidad logren en el siglo XXI dejar de lado los aspectos mecánicos o técnicos y aplicar el método científico en la Contabilidad aún con el riesgo de ser atacados, criticados o incluso ridiculizados; proponemos entonces intentar una "nueva era" de la Contabilidad.

Palabras clave: Contabilidad, metodología, abordajes científicos, teoría contable, reflexiones.

\section{Theoretical physicists debate and help accounting theorists}

Abstract: The present work reflects on the current state of accounting theory fundamentals and recognition to Max Planck, Albert Einstein and James Murphy on their methodological approaches for social sciences, in a reflection about the help they represent for accounting theorists. Regarding these approaches, we consider necessary that accounting theorists in the XXI century manage to leave aside mechanical or technical aspects and instead apply the scientific method to accounting, even with the risk of being attacked, criticized or even ridiculed. We thus propose trying a "new era" in accounting.

Keywords: Accounting, methodology, scientific approaches, accounting theory, reflections.

\section{Les physiciens théoriciens débatent et aident les théoriciens de la comptabilité}

Résumé: Cet article réfléchi sur l'état actuel des principes de la théorie comptable et sur la reconnaissance à Max Planck, Albert Einstein et James Murphy sur leurs approches méthodologiques en sciences sociales. Cette réflexion souligne l'aide que ces méthodes peuvent porter aux théoriciens de la comptabilité. Il est désormais nécessaire que les théoriciens de la comptabilité mettent de côté les aspects méchaniques ou téchniques et qu'ils commencent à appliquer la méthode scientifique à la comptabilité, même avec le risque d'être attaqués, critiqués ou même ridiculisés. Nous proposons donc ici d'inicier une nouvelle ère de la comptabilité.

Mots-clés: Comptabilité, méthodologie, approches scientifiques, théorie comptable, réflexions.

Os físicos teóricos debatem e ajudam aos teóricos da contabilidade

Resumo: $O$ presente trabalho reflete sobre o status atual dos fundamentos da teoria contábil e o reconhecimento a Max Planck, Albert Einstein e James Murphy sobre suas abordagens metodológicas para as ciências sociais, em uma reflexão sobre a ajuda que podem representar para os teóricos da contabilidade. Com relação a estes textos, consideramos necessário que os teóricos da contabilidade atinjam no século XXI abrir mão dos aspectos mecânicos ou técnicos e aplicar o método científico na contabilidade ainda com o risco de ser atacados, criticados ou até ridiculizados; a proposta é então tentar uma "nova era" da contabilidade.

Palavras chave: Contabilidade, metodologia, abordagens científicas, teoria contábil, reflexões. 
Cont. udea (enero-junio), pp. 29-38. (c) Universidad de Antioquia-2018.

\title{
Los físicos teóricos debaten y ayudan a los teóricos de la contabilidad
}

\author{
Carlos Luis García Casella \\ Doi: https://doi.org/10.17533/udea.rc.n72a02
}

Primera versión recibida en abril de 2018-Versión final aceptada en mayo de 2018

\section{Introducción}

I eyendo el libro "Orden y Misterio" de Komar (1996), encontré una Linteresante referencia a ciertas palabras del prólogo efectuado por Albert Einstein a la obra "A dónde va la ciencia" de Planck (1942) que me llamaron la atención:

a) "Por tratar con firmeza acerca de los hombres que se dedican a la ciencia privilegiando a los que lo hacen por el estado mental que proporciona en este caso el poder impulsor es semejante al del devoto o el amante, el esfuerzo largamente prolongado no es inspirado por un plan o propósito establecido, su inspiración surge de un hambre del alma." (Komar, 1996, p.140)

b) Señala asimismo que “(...) del elogio de Planck escrito por Einstein resulta claro la primacía de la ciencia desinteresada que brota del hambre interior del alma, apagado por el descubrimiento del orden profundo de lo real.” (Komar, 1996, p.140).

Busqué, entonces, el libro de Planck (1941) y encontré un conjunto de argumentos que me parecen adecuados para que los investigadores de Teoría Contable puedan inspirarse en la experiencia de dos teóricos de la física teórica para tratar de trasladar sus reflexiones a la contabilidad teórica.

A partir de lo anteriormente expuesto no pretendo colocarme a la altura de los descubridores de la teoría de los cuantos y de la teoría de la relatividad, pero pienso que es bueno leerlos si queremos mejorar la teoría contable en Argentina, en la región latinoamericana y en el mundo.

Y es aquí donde encontré el apoyo del colega Mattessich (2008) con su publicación sobre 200 años de investigación científica mundial en materia de teoría contable, quien en el capítulo 17 trata el tema de la investigación científica contable en la Argentina. Y con verguienza de aparente autoelogio 
transcribo lo que el mencionado autor expresa Mattessich (2008): "One of the prolific academics in Argentina is Garcia Casella 1997,1998, 1999a ,1999b, García Casella and Rodriguez de Ramírez 2000, 2001, 2002, 2004- Only to mention the more recent publications" (p.287).

Dejando de lado los elogios de Mattessich (2008) me parece que puedo intentar aprender a dónde va la ciencia para aplicarlo a la contabilidad con el apoyo de estos físicos teóricos. Los apartados que se exponen a continuación seguirán el orden del libro de Planck (1941).

\section{El libro de Max Planck con Albert Einstein y James Murphy}

El libro de Planck (1941) con Albert Einstein y James Murphy me deslumbró, ya que hacía tiempo que tenía mis dudas sobre las siguientes cuestiones:

a) No todos los hombres que se dedican a la ciencia lo hacen por amor a la ciencia misma, en nuestro caso, la ciencia de la contabilidad.

b) Un motivo para dedicarse a la investigación científica sería ensayar la construcción de una imagen que proporcione cierta expresión tangible de lo que la mente humana ve en la naturaleza (Planck, 1941); incluyendo en la naturaleza, la física y la social humana.

c) Hay límites al conocimiento humano.

d) El bosquejo biográfico de Planck (1941) hecho por James Murphy sirve para visualizar:

e) Las consecuencias de los nuevos descubrimientos.

f) Destacar las dificultades que encontró.

En los capítulos de su libro, Planck (1941) efectúa un análisis de 50 años de ciencia y el mencionado análisis incluye:

a) La teoría electrónica.

b) La teoría de la relatividad.

c) La teoría de los cuantos.

d) La imagen del universo físico en la ciencia.

e) Causalidad y libre albedrio.

f) La respuesta de la ciencia.

g) Desde lo relativo a lo absoluto.

h) Epílogo: Un dialogo socrático.

\section{El prólogo de Albert Einstein}

En el comienzo de su intervención Albert Einstein se dedica a clasificar a los investigadores de la ciencia y asimismo los evalúa; así dice:

Algunos hombres se dedican a la ciencia, pero no todos lo hacen por amor a la ciencia misma, hay algunos que entran en su templo porque se les ofrece la oportunidad de desplegar sus talentos particulares. Para esta clase de hombres la ciencia es una especie de deporte en cuya práctica hallan un regocijo, lo mismo que el atleta se regocija con la ejecución de sus proezas musculares. (Planck, 1941, p.9-11) 
A continuación, se exponen diez comentarios sobre la citada intervención de Einstein (Planck, 1941).

\section{a. Primer comentario}

Consideramos verdadera la crítica de Einstein para quienes, en cualquier ciencia, investigan sin amor a la respectiva ciencia y merecen ser calificados como atletas de la tarea de búsqueda de avance en el conocimiento, pero no como amantes del saber sea físico o sea contable. Prosigue Einstein:

$Y$ hay otro tipo de hombres que penetran en el templo para ofrendar su masa cerebral con la esperanza de asegurarse un buen pago. Estos hombres son científicos tan solo por una circunstancia fortuita que se presentó cuando elegían su carrera. Si las circunstancias hubieran sido diferentes podrían haber sido políticos o magníficos hombres de negocio. (Planck, 1941, p.9-11)

\section{b. Segundo comentario}

Consideramos que es acertada la calificación de aquellos que ingresan a la tarea de investigación con método científico principalmente o exclusivamente en la esperanza de asegurarse una remuneración alta; pero para Einstein sin amor a la ciencia misma su valor como investigador decae. Prosigue terminante Einstein:

Si descendiera un ángel del Señor y expulsara del templo de la ciencia a todos aquellos que pertenecen a las categorías mencionadas temo que el templo apareciera casi vacío. Pocos fieles quedarían, algunos de los viejos tiempos, algunos de nuestros días. Entre estos últimos se hallaría nuestro Planck. He aquí porque siendo tanta estima por él. (Planck, 1941, p.9-11)

\section{c. Tercer comentario}

Reconoce Einstein (Planck, 1941) que la mayoría de los investigadores no tienen el amor a la ciencia misma y de ese modo, esos pocos con ese amor parece que serían los que sostienen la investigación y logran más resultados en base a su amor a la ciencia. Según recapacita Einstein:

Me doy cuenta de que esa decisión significa la expulsión de algunas gentes dignas que han construido una gran parte, quizá la mayor, del templo de la ciencia, pero al mismo tiempo hay que convenir que si los hombres que se han dedicado a la ciencia pertenecieron tan sólo, a esas dos categorías, el edificio nunca hubiera adquirido las grandes proporciones que exhibe al presente, igual que un bosque jamás puede crecer si solo se compusiera de enredaderas. (Planck, 1941, p.9-11)

\section{d. Cuarto Comentario}

Aquí vemos que Einstein (Planck, 1941) reconoce la participación de esa "gente digna" que ha construido gran parte de los nuevos conocimientos, pero parece que lo han hecho con la conducción de amantes de la ciencia como Planck en Física; él llama "enredaderas" a los que investigan guiados por los amantes de la disciplina objeto de estudio como árboles fuertes del bosque 
del saber. Aquí Einstein trata sobre los posibles arboles fuertes del bosque que apoyan a la enredadera de este modo:

Pero olvidémonos de ellos: Non ragionam di lor y vamos a dirigir nuestras miradas a aquellos que merecieron el favor del ángel. En su mayor parte son gentes extrañas, taciturnas, solitarias. Pero a pesar de su mutua semejanza están muy lejos de ser iguales a los que nuestro hipotético ángel expulsó. (Planck, 1941, p.9-11).

e. Quinto comentario

Comienza la descripción de los investigadores a la manera de Planck (1941), ya que parecen ser personas diferentes de los otros investigadores; resulta que su manera de ser extraña, taciturna y solitaria los hace amantes apasionados de la disciplina que estudian. En el párrafo siguiente Einstein (Planck, 1941) trata de describir su manera de ser así:

¿Qué es lo que les ha conducido a dedicar sus vidas a la persecución de la ciencia? Difícil es responder a esta cuestión, y puede que jamás sea posible dar una respuesta categórica. Me inclino a aceptar con Schopenhauer que uno de los más fuertes motivos que conduce a las gentes a entregar sus vidas al arte o a la ciencia es la necesidad de huir de la vida cotidiana con su gris y fatal pesadez y así desprenderse de las cadenas de los deseos temporales que se van suplantando en una sucesión interminable, en tanto que la mente se fija sobre el horizonte del medio que nos rodea día tras día. (Planck, 1941, p.9-11)

\section{f. Sexto Comentario}

Ante las grandes dificultades de establecer porqué alguien se dedica a investigar, sea física o contabilidad teórica, recurre a un motivo negativo: Huir de la vida cotidiana según Schopenhauer. Pero añade otro motivo positivo Einstein, así:

Pero a este motivo negativo debe añadirse otro positivo. La naturaleza humana ha intentado siempre formar por sí misma una simple y simpática imagen del mundo circundante. En consecuencia, ensaya la construcción de una imagen que proporcione cierta expresión tangible de lo que la mente ve en la naturaleza. (Planck, 1941, p.9-11)

\section{g. Séptimo Comentario}

En el caso de los investigadores de la ciencia social denominada Contabilidad, el motivo positivo para investigar, es lograr construir una imagen de la realidad de la actividad humana en sociedad llamada Contabilidad, en todo lo que rodea al sujeto investigador: Como indicamos García y Rodríguez (2001). Así podemos enunciar algunos de los elementos incluidos en su dominio:

1. Informes Contables de uso externo a los emisores.

2. Personas y grupos de personas emisoras de los diversos informes contables.

3. Personas revisoras que opinan sobre la calidad de los informes contables.

4. Personas o grupos de personas usuarias o destinatarias de los diversos informes contables. 
5. Personas o grupos de personas reguladoras de los distintos informes contables.

6. Microsistemas contables propios de cada ente.

7. Macro sistemas contables definidos en ciudades, países, regiones, o en tipos de actividad o en clases diferentes de organizaciones.

8. Modelos contables necesarios para determinar variables relevantes en diversas situaciones.

9. Informes contables de uso interno en cada ente.

10. Informes contables de organismos gubernamentales.

11. Informes contables macroeconómicos.

12. Informes contables macrosociales.

13. Informes contables microsociales.

14. Segmentos contables.

Luego prosigue Einstein, aclarando temas de física teórica que se pueden aplicar a la contabilidad teórica (Planck, 1941): "Por otra parte, el físico tiene que ser severo y abnegado respecto al material que utiliza" (p. 11). Además, añade:

Debe contentarse con reproducir los más simples procesos que se ofrecen a nuestra experiencia sensorial, pues los procesos más complejos no pueden ser representados por la mente humana con la sutil exactitud y la secuencia lógica que son indispensables para el físico teórico. (Planck, 1941, p.11)

\section{h. Octavo Comentario}

La aplicación a la teoría contable o contabilidad teórica exige:

a) Severidad

b) Abnegación

Con el material de la realidad físico-espiritual de la actividad contable y la posibilidad de su comprensión, no se ven con exactitud matemática ni con una secuencia lógica y entonces buscará una respuesta. A modo de respuesta expone Einstein:

Incluso a expensas de la amplitud tenemos que asegurar la pureza, la claridad y la exacta correspondencia entre la representación y la cosa representada. Al darnos cuenta de que es muy pequeña la parte de la naturaleza (Digo yo físico-espiritual) que así podemos comprender y expresar en una formula exacta, mientras tiene que ser excluido todo lo más sutil y complejo, es natural preguntarse: ¿Qué tipo de atracción puede ejercer esta obra? ¿Merece el pomposo nombre de imagen del mundo (digo yo físico-espiritual) el resultado de una selección tan limitada?

Creo que sí, pues las leyes más generales sobre las cuales se construye la estructura mental de la Física Teórica tienen que ser derivadas estudiando en la naturaleza incluso los fenómenos más sencillos. (Planck, 1941, p.9-11) 


\section{i. Noveno Comentario}

En la Contabilidad también hace falta buscar la correspondencia entre la realidad y entonces también se verifica que es muy poca la parte de realidad que podemos expresar en forma exacta y la solución que podemos aplicar, es encontrar leyes generales estudiando los fenómenos sencillos.

En este aspecto en el libro de García y Rodríguez (2001) propusimos leyes para ocho problemas fundamentales de la Contabilidad:

a) 16 leyes sobre el dominio o universo del discurso contable (p. 14-15).

b) 11 leyes sobre la naturaleza o status epistemológico de la contabilidad (p.69-70).

c) 16 leyes sobre las relaciones de la contabilidad con otras disciplinas (p.125126).

d) 9 leyes sobre la segmentación o unidad contable absoluta (p.145-146).

e) 13 leyes sobre los sistemas contables (p. 171-172).

f) 10 leyes sobre medición (p. 197-198).

g) 5 leyes sobre personas o sujetos de la actividad contable (p.265).

h) 5 leyes sobre modelos en la teoría general contable (p. 281).

Futuras investigaciones reducirían la cantidad de leyes o regularidades de la Contabilidad.

Einstein (Planck, 1941) sigue desarrollando sobre esos fenómenos sencillos y dice:

Si son bien conocidos, hay que ser capaz de deducir de ellos, mediante el razonamiento puramente abstracto, la teoría de todos los procesos de la naturaleza, incluyendo los de la vida misma. He querido decir teóricamente pues en la práctica tal proceso de deducción está mucho más allá de la capacidad del razonamiento humano. Por tanto, el hecho de que en la ciencia tengamos que contentarnos con una imagen incompleta del universo físico no es debido a la naturaleza del universo sino más bien a nosotros mismos. (Planck, 1941, p.9-11)

\section{j. Decimo Comentario}

Creemos que en contabilidad teórica tendremos una imagen incompleta del universo físico-espiritual que analizamos por las limitaciones de la capacidad del razonamiento humano. Y ahora llega el momento del descubrimiento por parte de Einstein de las leyes elementales más generales de este modo:

Así la labor suprema del físico es el descubrimiento de las leyes elementales más generales a partir de las cuales puede ser deducida la imagen del mundo. Pero no existe un camino lógico para el descubrimiento de esas leyes elementales. Existe únicamente la virtud de la intuición ayudada por un sentido para el orden que yace tras de las apariencias y este Einfuehlung se desarrolla por la experiencia. (Planck, 1941, p.12)

Aplicado lo anterior a la contabilidad, opinamos:

a) La tarea del teórico contable es ir descubriendo las leyes o regularidades elementales lo más generales posibles. 
b) En base a ello se deduce la imagen del universo del discurso contable.

c) No existe un camino lógico para esa tarea y el teórico contable deberá usar la virtud de la intuición para lograr encontrar el orden en la vida social vinculada a la ciencia contabilidad.

d) La experiencia es la ayuda que nos propone Einstein: un investigador $\sin$ experiencia fracasaría igual que si fuera sin intuición.

Y termina su intervención Einstein destacando lo importante para el contable teórico:

I. “(...) la síntesis conceptual que es un trasunto del mundo empírico puede ser reducida a unas cuantas leyes fundamentales sobre las cuales se construye lógicamente toda la síntesis.” (Planck, 1941, p.12).

II. "Y esto no puede ser referido al trabajo mental del físico (o contable agregado nuestro). Sino una cualidad que es inherente al mundo de la percepción, Leibniz expresó adecuadamente esta cualidad denominando la armonía preestablecida." (Planck, 1941, p.12).

Opinamos que eso se debe buscar en la contabilidad y su universo social.

III. Siguiendo a Einstein: “(...) el estado mental que proponemos en este caso el poder impulsor es semejante al del devoto o al del amante. El esfuerzo prolongado no es inspirado por un plan o propósito establecido. Su inspiración surge de un hambre del alma." (Planck, 1941, p.12)

\section{El bosquejo biográfico de Max Planck por J. Murphy}

a) El encuentro entre Murphy y Einstein

Entre las páginas 14 y 38 de Planck (1941), James Murphy establece una pequeña biografía de Max Planck. Primero recuerda su entrevista de junio de 1932 al visitar a Albert Einstein cerca de Berlín y el momento del diálogo donde:

a) Surgieron los diversos problemas filosóficos a los que la teoría de los cuantos había dado lugar.

b) Parece que concluyeron con que la teoría de los cuantos ha sido una fuerza más fundamental para provocar la revolución moderna del pensamiento científico, aunque la teoría de la relatividad habría apresado el interés del mundo.

c) Consideramos que es adecuado analizar la obra del teórico físico Planck (1941) para aprovechar su lucha y su éxito científico y sacar conclusiones para los teóricos de la contabilidad en el país, en Latinoamérica y en el mundo.

b) El papel de Max Planck en la ciencia física

Para indicar el papel de Planck (1941), Murphy cita a Sir James Jean en su libro "EL universo misterioso", en el mismo se señala:

A finales del siglo XIX fue por primera vez posible estudiar el comportamiento de la molécula, del átomo y del electrón. El siglo se ha prolongado suficientemente para que 
García, C. Los físicos teóricos debaten y ayudan a los teóricos de la contabilidad

la ciencia descubriera que ciertos fenómenos, la radiación y la gravitación en particular, necesitan a todos los ensayos de explicación puramente mecánica.

Mientras los filósofos están aún discutiendo si puede ser construida una máquina para reproducir los pensamientos de Newton, la sensibilidad de Bach o la inspiración de Miguel Ángel, el tipo medio de hombre de ciencia se ha convencido rápidamente de que es imposible construir máquinas que reproduzcan la luz de una bujía o la caída de una manzana.

Entonces, en los últimos meses de la centuria pasada, el profesor Max Planck, de Berlín, realizó una tentativa para explicar ciertos fenómenos de radiación que hasta aquel día no habían podido ser interpretados. Su explicación no solo era nomecánico en su naturaleza sino también parecía imposible relacionarla con cualquier pensamiento inspirado en el terreno de la mecánica. Por esta razón fue grandemente criticado, atacado e incluso ridiculizado. Pero Planck demostró brillantemente su exactitud y luego la desarrolló en la moderna teoría de los cuantos, que constituye uno de las principales dominantes de la física moderna. También, aunque esto no se llegó a comprender en aquel momento, su hallazgo marca el fin de la era mecánica de la ciencia y la aparición de una nueva era.

\section{Reflexión final}

Frente a estos textos, consideramos necesario que los teóricos de la Contabilidad logren en el siglo XXI dejar de lado los aspectos mecánicos o técnicos y aplicar el método científico en la Contabilidad aún con el riesgo de ser:

a) Atacados;

b) Criticados;

c) Incluso ridiculizados.

Proponemos entonces intentar una "nueva era" de la contabilidad.

\section{Referencias bibliográficas}

García, C. L. y Rodríguez, M. C. (2001). Elementos para una Teoría General de la Contabilidad. Buenos Aires: Editor La Ley S.A. (ISBN 950-527-480-7) 305 páginas, junio.

Komar, M. (1996) Fe y cultura el artículo 41 del documento La escuela Católica en Komar, M. En Orden y Misterio (177 páginas). Buenos Aires, Argentina: Emecé Editores. 1a edición Febrero Rosario República Argentina (ISBN 950-04-1310-0) 201.337 Las palabras de Albert Einstein (pág. 138/140).

Mattessich, R. (2008) Two Hundred Years of Accounting Research an International survey of personalities, ideas and publications (From the beginning of the nineteenth century to the beginning of the twenty-first century) Park Square, Milton Park, Abingdon Oxon, Estados Unidos: Routledge 2 OX144RN. 590 páginas (ISBN 0-203-93985-9 Master e-book ISBN).

Planck, M. (1941) ¿A dónde va la Ciencia? prólogo de Albert Einstein; introducción biográfica de James Murphy. Buenos Aires, Argentina: Editorial Losada S.A. Traducción de Felipe Jiménez de Asúa, 242 páginas. 